\title{
Design and Optimization of a New Composite Thermal Insulating Fluorocarbon Coating
}

\author{
Xiu-Fang YE, Dong-Chu CHEN*, Jing-Song ZHANG, Xiao-Ting Wang, Meng- \\ Lei CHANG and Xiao-Yan HUANG
}

School of Materials Science and Energy Engineering Foshan University, Foshan, China

Email: yexf@fosu.edu.cn, chendc@fosu.edu.cn, 2592484235@qq.com,1441209914@qq.com, 26009823@qq.com, 345932002@qq.com

\section{Keywords- thermal insulation; composite; response surface methodology; optimization}

\begin{abstract}
Based on the mechanism of thermal insulation, a new composite fluorocarbon thermal insulation coating (FTIC) was prepared with three different fillers of closed pore perlite, hollow glass microsphere and nano-antimony tin oxide (ATO) powder, in order to get the best thermal insulation performance, the formula of the composite FTIC was optimized by varying three independent parameters (the content of closed pore perlite, hollow glass microsphere and nanoATO powder) and using a central composite design under response surface methodology. The test results show that, the temperature difference for the optimized FTIC system is $8.8^{\circ} \mathrm{C}$, which is much the same to the value of $9.0^{\circ} \mathrm{C}$ predicated by response surface methodology model.
\end{abstract}

\section{Introduction}

Considering thermal insulation coating is one of the effective ways to improve building insulation properties and to reduce the energy consumption on air conditioning, we have done much in this area [1-7]; however, most researches focus on single or just two insulation mechanisms of thermal insulation combined together. In fact, the heat transfer of building is a result of combined action including obstruction, radiation and reflection. As a result, excellent thermal insulation coatings that can be used on building are still lacking in China. So, the multiple models and synergistic mechanism of thermal insulation coating system should be further studied in order to get excellent thermal insulation coatings.

To meet the national needs for energy saving and emission reduction, this paper prepared a new fluorocarbon thermal insulation coating (FTIC) based on polyvinylidene fluoride emulsion. Using the principle of a central composite design under response surface methodology, this study has considered the content of three different functional fillers as the factors that could be related to thermal insulation performance of FTIC. Based on these, a new composite FTIC with multiple thermal insulation mechanism including obstruction, reflection and radiation was prepared. And the formulation of the composite FTIC was optimized by response surface methodology experiment.

\section{Experimental Methods}

\section{Coating Preparation}

The composite fluorocarbon thermal insulation coating (FTIC) is prepared by mixing the polyvinylidene fluoride emulsion, thermal insulation functional fillers (as closed pore perlite for obstructive, hollow glass microsphere for reflective and nano-antimony tin oxide (ATO) powder for radiative coatings), solvents and additives according to a certain proportion seen in Table 1 according to our earlier work[8] and the preparation process is as shown in Fig.1. 
Table 1 The formula of composite fluorocarbon thermal insulation coating

\begin{tabular}{ccc}
\hline & Materials & Contents $(\mathrm{g})$ \\
\hline Base & Polyvinylidene fluoride & $30-40$ \\
Solvents & Isophorone & $10-20$ \\
& Dimethylbenzene & $5-10$ \\
Additives & Silane coupling agent & $1-2$ \\
Thermal & BYK - 141 & $1-3$ \\
insulation & Closed pore perlite & \\
functional & Hollow glass microsphere & $0.2-5$ \\
fillers & nano-ATO powder & \\
\hline
\end{tabular}

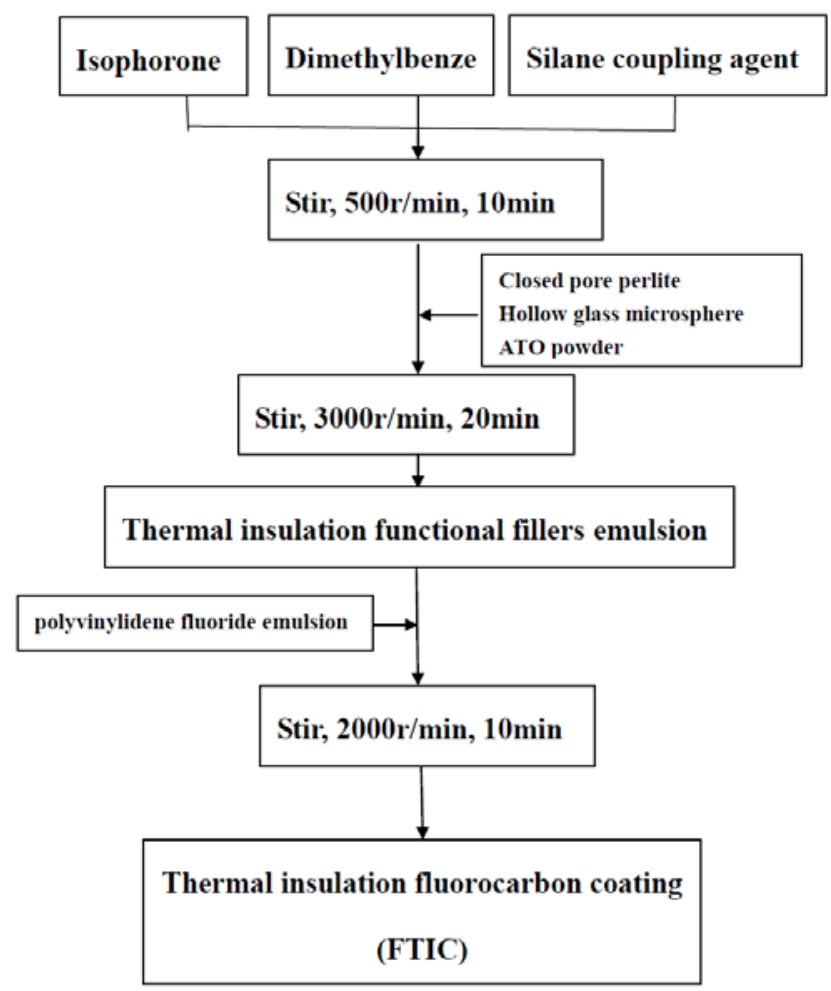

Fig.1 Process flowchart of FTIC preparation

\section{Thermal Insulation Performance Measurements}

The thermal insulation performance of FTIC was measured by a self-developed lamp illumination apparatus, which is built according to the "reflective building insulation coatings" (JG/T 235-2008). The FTIC with different categories/content of functional fillers was applied onto cleaned and dried $80 \times 80 \times 2 \mathrm{~mm}$ aluminium panel. The obtained dry coating thickness for these coatings was approximately $0.5 \mathrm{~mm}$. Meanwhile, ordinary PVDF coatings was also applied onto cleaned and dried $80 \times 80 \times 2 \mathrm{~mm}$ aluminium panel and dried as the sample for blank test. Details of the device and its working principle are described elsewhere [9-13], the relative temperature difference between the surface and inside after heating balance is recorded as $\Delta \mathrm{T}$. After heating balance, the temperature differences between the surface and inside are recorded as $\Delta \mathrm{T}_{\mathrm{t}}$ for FTIC and $\Delta \mathrm{T}_{\mathrm{o}}$ for ordinary coating correspondingly. Accordingly, the absolute temperature difference of the FTIC is $\mathrm{T}_{\mathrm{d}}\left(T d=\Delta T_{t}-\Delta T_{o}\right)$, which represents the absolute heat insulation effect of the test sample. 


\section{Results and Discussion}

\section{Single Factor Experiments of the Three Functional Fillers}

This study has considered the content of three different functional fillers as the factors that could be related to thermal insulation performance of FTIC. Single factor experiment method was adopted to investigate the influence of functional fillers on thermal insulation performance of FTIC. Closed pore perlite, hollow glass microsphere and nano-ATO powder were used respectively to prepare obstructive coating, reflective coating and radiative coating, and the thermal insulation performance of these three categories of FTIC were studied in our earlier work, based on the data we get from our earlier work [8], we created the variation rules of absolute temperature difference based on the category of functional fillers as shown in Fig.2.

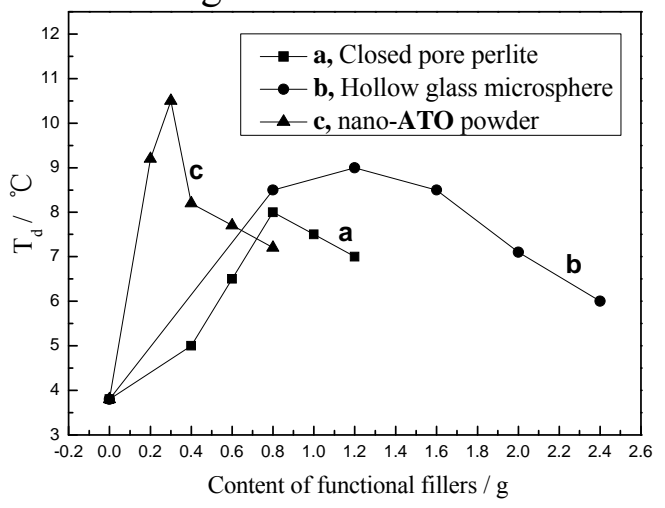

Fig.2 Variation rules of absolute temperature difference based on the category of functional fillers

The results showed that the category and content of functional fillers can both affect the thermal insulation performance of FTIC. For all three functional fillers, the temperature difference is not always increase when more content of functional fillers was added in, but there is a maximum temperature difference showed in the experiment range.

\section{Formula Optimized by Response Surface Methodology}

Based on the earlier experimental results, the category and content of functional fillers were considered significant. Thereafter, the exact optimal values for the individual factors were determined using a full-factorial central composite design, taking the content of the three functional fillers as the variation, a three-factor three-level experiments design was employed as shown in Table 2 and Table3. The actual values of temperature difference for the variables at various levels are given in Table3. The full-factorial central composite design for three experimental variables, each at three levels required 17 experiments to be performed. The effect of each variable, sum of squares, mean square, F-value, P-value and confidence level (\%) were determined using the statistical software package Design-Expert Software8.05b.

Table 2 Factors and levels in response surface design

\begin{tabular}{lrrrc}
\hline \multirow{2}{*}{ Variables } & \multicolumn{3}{c}{ Values } & \multirow{2}{*}{ Code value } \\
\cline { 2 - 4 } & -1 & 0 & +1 & \\
\hline Closed pore perlite $(\mathrm{g})$ & 0.6 & 0.8 & 1.0 & $\mathrm{~A}$ \\
Hollow glass microsphere $(\mathrm{g})$ & 0.8 & 1.2 & 1.6 & $\mathrm{~B}$ \\
nano-ATO powder(g) & 0.2 & 0.3 & 0.4 & $\mathrm{C}$ \\
\hline
\end{tabular}


Table 3 The design of response surface methodology experiments and the corresponding temperature difference of ftic samples

\begin{tabular}{ccccc}
\hline \multirow{2}{*}{ RUN } & \multicolumn{3}{c}{ Variables/Levels } & \multirow{2}{*}{ Response $\left(\mathrm{T}_{\mathrm{d}}\left({ }^{\circ} \mathrm{C}\right)\right)$} \\
\cline { 2 - 4 } & $\mathrm{A}$ & $\mathrm{B}$ & $\mathrm{C}$ & 6.0 \\
\hline 1 & 1 & 0 & -1 & 7.0 \\
2 & 1 & -1 & 0 & 8.5 \\
3 & -1 & -1 & 0 & 9.0 \\
4 & 0 & 0 & 0 & 8.5 \\
5 & 1 & 1 & 0 & 7.5 \\
6 & -1 & 0 & -1 & 9.0 \\
7 & 0 & 0 & 0 & 7.5 \\
8 & 1 & 0 & 1 & 7.0 \\
9 & 0 & 1 & 1 & 7.5 \\
10 & 0 & -1 & -1 & 7.0 \\
11 & 0 & -1 & 1 & 7.0 \\
12 & -1 & 0 & 1 & 9.0 \\
13 & 0 & 0 & 0 & 8.0 \\
14 & 0 & 1 & -1 & 9.0 \\
15 & 0 & 0 & 0 & 9.0 \\
16 & 0 & 0 & 0 & 7.5 \\
17 & -1 & 1 & 0 & \\
\hline
\end{tabular}

Table 3 represents the experimental design and results obtained for the novel composite thermal insulating fluorocarbon coating. The analysis results of variance on the temperature difference of FTIC are represented in Table 4. Data were best fitted by the following quadratic polynomial equation: $\mathrm{Y}=9.00-0.19 \mathrm{~A}+0.13 \mathrm{~B}-0.063 \mathrm{C}+0.62 \mathrm{AB}+0.50 \mathrm{AC}-0.13 \mathrm{BC}-0.75 \mathrm{~A}^{2}-0.38 \mathrm{~B}^{2}-1.25 \mathrm{C}^{2}$, where $\mathrm{Y}$ is the predicted response $\left(\mathrm{T}_{\mathrm{d}}\right)$, and $\mathrm{A}, \mathrm{B}$, and $\mathrm{C}$ are the coded values for the content of closed pore perlite, hollow glass microsphere and nano-ATO powder, respectively.

Table 4 The analysis results of variance by central composite quadratic model

\begin{tabular}{cccccc}
\hline Source & Sum of squares & Degree of freedom & Mean square & F value & P $>$ F \\
\hline Model & 13.45 & 9 & 1.49 & 12.88 & 0.0014 \\
A-A & 0.28 & 1 & 0.28 & 2.42 & 0.1635 \\
B-B & 0.13 & 1 & 0.13 & 1.08 & 0.3339 \\
C-C & 0.031 & 1 & 0.031 & 0.27 & 0.6198 \\
AB & 1.56 & 1 & 1.56 & 13.46 & 0.0080 \\
AC & 1.00 & 1 & 1.00 & 8.62 & 0.0219 \\
BC & 0.063 & 1 & 0.063 & 0.54 & 0.4869 \\
A $^{2}$ & 2.37 & 1 & 2.37 & 20.40 & 0.0027 \\
B $^{2}$ & 0.59 & 1 & 0.59 & 5.10 & 0.0585 \\
C $^{2}$ & 6.58 & 1 & 6.58 & 56.68 & 0.0001 \\
Residual & 0.81 & 7 & 0.12 & & \\
Lack of Fit & 0.81 & 3 & 0.27 & & \\
Pure Error & 0.000 & 4 & 0.000 & & \\
Corrected Total & 14.26 & 16 & & & \\
\hline
\end{tabular}

In order to validate the regression coefficient, an analysis of variance on temperature difference of FTIC was performed (Table 4). Values of "P $>F$ " less than 0.0001 indicate model terms are significant. In this case, $\mathrm{AB}, \mathrm{AC}, \mathrm{A}^{2}, \mathrm{C}^{2}$ are significant model terms, they contributed a significant effect on the temperature difference of FTIC as seen in Table 4. It is noteworthy that, Greater Fvalue from unity made it more certain that the factors explained adequately the variations in the data 
about its means, and the estimated factor effects are real. Fig. 3 shows the iso-response 3D surface plots, showing the effect of closed pore perlite, hollow glass microsphere and nano-ATO powder at different contents on the temperature difference of FTIC. The statistical optimal values for the variables were obtained by moving along the major and minor axis of the contour, and the response at the center point yielded the maximum temperature difference. As a result, based on the 3D plots, the optimal concentration values for $\mathrm{A}, \mathrm{B}$, and $\mathrm{C}$ (closed pore perlite, hollow glass microsphere and nano-ATO powder) were identified as $0.8,1.2$, and $0.3 \mathrm{~g}$, respectively, so, the formula of the FTIC optimize by response surface methodology is as seen in Table4, and the maximum temperature difference can be up to $9.0^{\circ} \mathrm{C}$ as predicated by the model, which is much higher than the temperature difference of FTIC with just one single mechanism functional fillers[8].

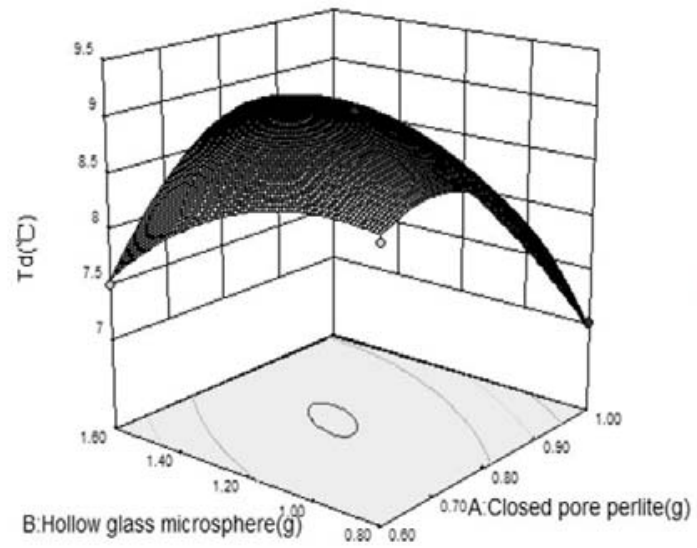

(a) Diagrams of $\mathrm{AB}$ on $\mathrm{T}_{\mathrm{d}}$

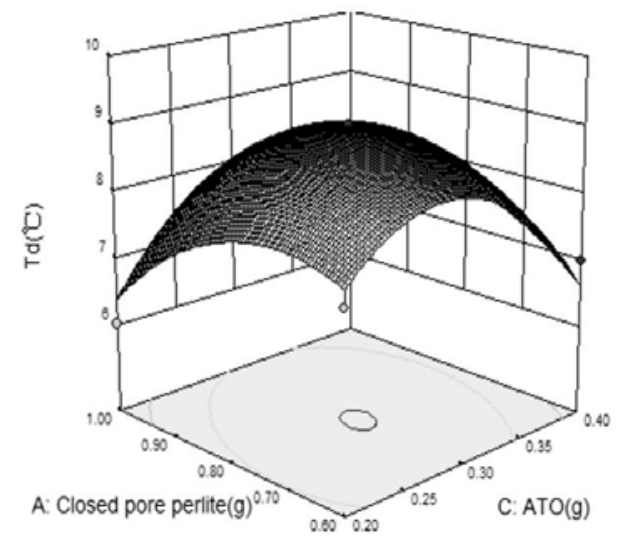

(b)Diagrams of $\mathrm{AC}$ on $\mathrm{T}_{\mathrm{d}}$

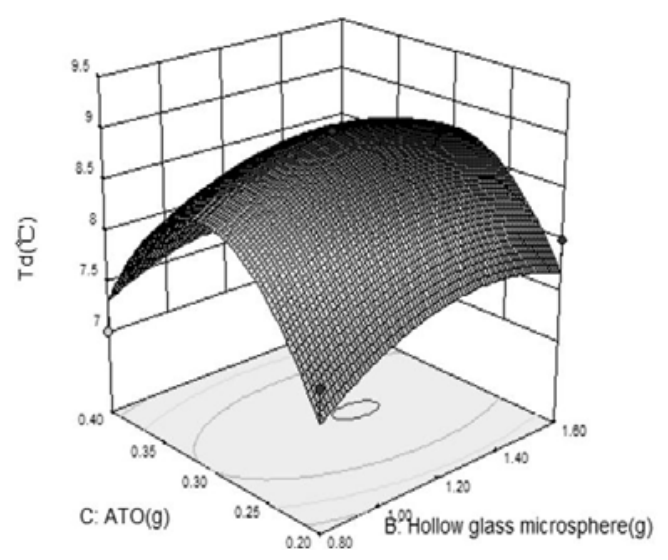

(c) Diagrams of BC on $\mathrm{T}_{\mathrm{d}}$

Fig.3 Analysis of variance for full-factorial central composite quadratic model 
Table 5 The formula of the composite FTIC

\begin{tabular}{ccc}
\hline & Materials & Contents $(\mathrm{g})$ \\
\hline Base & Polyvinylidene fluoride & $30-40$ \\
Solvents & Isophorone & $10-20$ \\
& Dimethylbenzene & $5-10$ \\
Additives & Silane coupling agent & $1-2$ \\
& BYK - 141 & $1-3$ \\
Functional & Closed pore perlite & 0.8 \\
fillers & Hollow glass microsphere & 1.2 \\
& nano-ATO powder & 0.3 \\
\hline
\end{tabular}

\section{Confirmatory Test of the Model}

Based on the research done above, the formula of a novel composite FTIC system was optimization by response surface methodology. For the novel composite FTIC, three different categories of functional fillers were contained, and as a result, it can promote the collaborative thermal insulation effect including obstruction, radiation and reflection, the absolute temperature difference $\mathrm{Td}$ could be up to $9.0^{\circ} \mathrm{C}$ as predicated. Thus, in order to verify this prediction, a composite FTIC was prepared according to the formula seen in Table 5. The absolute temperature difference $\mathrm{Td}$ was tested, and the result showed that the absolute temperature difference $\mathrm{Td}$ is $8.8^{\circ} \mathrm{C}$, which is in great agreement with the results predicated by response surface methodology.

\section{Conclusions}

(1) In the formula of a novel composite FTIC optimized by the response surface methodology, the contents of closed pore perlite, hollow glass microsphere and nano-ATO powder are $0.8,1.2$, and $0.3 \mathrm{~g}$, respectively.

(2) The test result showed that the absolute temperature difference of the optimized FTIC is $8.8^{\circ} \mathrm{C}$, which is much higher than the absolute temperature difference of the FTIC with just one single mechanism functional filler.

(3) For the absolute temperature difference of the optimized FTIC, the predicted value $9.0^{\circ} \mathrm{C}$ is in great agreement with the results of tested value $8.8^{\circ} \mathrm{C}$, that is to say, the response surface methodology is very effective in formula optimization.

\section{Acknowledgment}

This work has been supported by Foundation for Distinguished Young Talents in Higher Education of Guangdong, China (2015KQNCX178), Green Building Materials and New Technology for Energy Conservation and Emissions Reduction Engineering Technology Research Center of Foshan and Laboratory Open Fund of Foshan University.

\section{References}

[1] L. Wang, J. Hang, L. Shi, X. Sun, F. Xu, "Preparation and characterization of NIR cutoff antimony doped tin oxide/hybrid silica coatings", Materials Letters, Vol.87, pp.35-38, 2012.

[2] K. Lu, Z. Ji, Z. Kong, H. Li, J. Zhang, "Preparation and thermal insulating properties of antimony doped nano-SnO2/waterborne polyurethane composite coatings", Journal of Inorganic Materials, Vol.27, pp.1117-1120,2012.

[3] Y. Shi, Z. Song, W. Zhang, J. Song, J. Qu, Z. Wang, Y. Li, L. Xu, J. Lin, "Physicochemcial properties of dirt-resistant cool white coatings for building energy efficiency", Solar Energy Materials \&Solar Cells, Vol.110, pp.133-139, 2013. 
[4] H. Shen, H.W. Tan, A. Tzempelikosa, "The effect of reflective coatings on building surface temperatures, indoor environment and energy consumption-an experimental study", Energy and Buildings, Vol.43, pp.573-580, 2011.

[5] Z. Dai, Z. Li, L. Li, G. Xu, "Synthesis and thermal properties of antimony doped tin oxide/waterborne polyurethane nanocomposite films as heat insulting materials", Polymers for Advanced Technologies, Vol.22, pp.1905-1911, 2011.

[6] Y.F. Liao, B.R. Zeng, M. Chen, S.J. Lin, H.J. Deng, J.T. Wu, Y.T. Xu, L.Z. Dai, "Preparation and properties of heat reflective insulation coatings", Polymer Materials Science and Engineering, Vol.28, pp.121-124, 2012.

[7] J.G. Li, "Preparation and application of elastic and hydrophobic non-bead type architectural reflective heat insulation coating”, Paint \& Coatings Industry, Vol.43, pp.1-5, 2013.

[8] X.F. Ye, D.C. Chen, M.L. Chang, Q.H. Liang, Q.P. Lu, "The preparation of fluorocarbon thermal insulation coating with different fillers", Advanced Materials Research, Vol. 1101,pp.3639,2015 .

[9] W. Guo, X. Qiao, Y. Huang, M. Fang, X. Han, "Study on energy saving effect of heatreflective insulation coating on envelopes in the hot summer and cold winter zone". Energy and Buildings, Vol.50, pp.196-203, 2012.

[10]J. Qu, J.R. Song, J.Q, Z.N. Song, W.D. Zhang, Y.X. Shi, T. Zhang, H.Q. Zhang, R.P. Zhang, Z.Y. He, X. Xue, "Transparent thermal insulation coatings for energy efficient glass windows and curtain walls", Energy and Buildings, Vol.77, pp.1-10, 2014.

[11]Z. Song, W. Zhang, Y. Shi, J.SONG, J. Qu, T. Zhang, Y. Li , H. Zhang, R. Zhang, “Optical properties across the solar spectrum and indoor thermal performance of cool white coatings for building energy efficiency”, Energy and Buildings, Vol.63, pp.49-58, 2013.

[12]X.F. Ye, L.Z. Cui, Y.H. Gao and Y.H. Yuan, "Development of waterborne composite acrylic thermal insulation exterior wall coatings", Paint\& Coatings Industry, Vol. 41,pp.51-53,2011.

[13]X.F. Ye, D.C. Chen, Q.H. Liang, F.Y. Li and S.P. Liang, "Preparation of energy-saving composite fluorocarbon heat-insulation coatings", Modern Chemical Industry, Vol.34, pp.9092,2014 . 